\title{
A novel immunotherapeutic modality with direct hemoperfusion targeting transforming growth factor-ß prolongs the survival of tumor-bearing rats
}

\author{
YOSHIKI YAMAMOTO ${ }^{*}$, YUJI UEDA ${ }^{1 *}$, TSUYOSHI ITOH $^{1}$, ARIHIRO IWAMOTO $^{1}$, \\ HISAKAZU YAMAGISHI ${ }^{1}$, MASAAKI SHIMAGAKI ${ }^{2}$ and KAZUO TERAMOTO ${ }^{2 *}$
}

\author{
${ }^{1}$ Department of Surgery, Division of Digestive Surgery, Graduate School of Medical Science, Kyoto Prefectural \\ University of Medicine, 465 Kajii-cho, Kawaramachi-Hirokoji, Kamigyo-ku, Kyoto 602-8566; ${ }^{2}$ Special Material \\ Research Laboratories, Toray Industries, Inc., 2-1 Sonoyama 3-chome, Otsu, Shiga 520-0842, Japan
}

Received July 24, 2006; Accepted August 24, 2006

\begin{abstract}
Immune responses are frequently depressed in patients with cancer. One of the reasons for a poor immune response is the presence of increased levels of immunosuppressive substances associated with tumor growth. Transforming growth factor- $\beta$ (TGF- $\beta$ ), a representative immunosuppressive cytokine, plays various roles in the progression of cancer. To remove immunosuppressive substances from tumor-bearing hosts, we developed an immunosuppressive substance adsorption (ISA) column for direct hemoperfusion (DHP) treatment. It is filled with extrafine fibers that can adsorb TGF- $\beta$. In this study, we investigated the effects of this DHP treatment on serum levels and activities of TGF- $\beta$, cellular immune responses, and anti-tumor effects in KDH-8 (TGF-ß-producing hepatocellular carcinoma cell line)-bearing rats. We further studied the ability of ISA fibers to adsorb tumor-associated immunosuppressive cytokines [TGF- $\beta$, interleukin (IL)-6 and vascular endothelial growth factor (VEGF)] in samples of body fluids obtained from patients with metastatic cancer. DHP treatment decreased serum levels and activities of TGF- $\beta$ in tumor-bearing rats and restored $\mathrm{T}$ lymphocyte response to mitogen. Tumor growth in rats treated by DHP was significantly slower than that in untreated rats. The survival time of treated rats was significantly longer than that of untreated rats. The concentrations of TGF- $\beta$, IL- 6 , and VEGF in the samples of human
\end{abstract}

Correspondence to: Dr Yuji Ueda, Department of Surgery, Division of Digestive Surgery, Graduate School of Medical Science, Kyoto Prefectural University of Medicine, 465 Kajii-cho, Kawaramachi-Hirokoji, Kamigyo-ku, Kyoto 602-8566, Japan

E-mail: yueda@koto.kpu-m.ac.jp

${ }^{*}$ Contributed equally

Key words: direct hemoperfusion, TGF- $\beta$, cytokine, immunosuppression, cancer immunotherapy body fluids were decreased markedly by in vitro treatment with ISA fibers. These results suggest that DHP treatment with an ISA column, which removes TGF- $\beta$ and other immunosuppressive substances from the sera of tumor-bearing hosts, is potentially a new immunotherapeutic strategy for cancer.

\section{Introduction}

Reversal of tumor-induced immunosuppression has been tried and considered a promising, less toxic approach to cancer therapy. Tumor-induced immunosuppression is orchestrated by several cytokines, including interleukin (IL)-6 (1), IL-10, prostaglandin (PG) E2, vascular endothelial growth factor (VEGF) (2), and transforming growth factor (TGF)- $\beta$. These cytokines have suppressive effects on immune effector cells, as well as on antigen-presenting cells. They also have tumor promoting effects, and their overexpressions have been associated with poor outcomes (6). Despite recent progress in tumor immunotherapy, currently available regimens have failed to meet initial expectations, partially because of tumor-induced immunosuppression in the host. Removal of these immunosuppressive factors by extracorporeal circulation appears promising and has been attempted by many investigators $(5,7)$.

Numerous studies have reported that plasma exchange treatment can enhance response to tumor immunotherapy and patients' quality of life (8-10). Israel et al reported that a single session of plasma exchange treatment produced a partial objective response with cancer regression in 8 of 27 patients with disseminated cancer who received no other treatments $(11,12)$. However, plasma exchange treatment has several drawbacks, including the risk of infectious diseases being transmitted by plasma transfusion and high costs.

Previous studies have reported the development of a Toraymyxin $^{\text {TM }}$ column designed to adsorb bacterial endotoxins for hemoperfusion therapy in patients with serious infectious diseases (13). We drew on this experience and developed novel immunosuppressive substance adsorption (ISA) fibers for anti-tumor hemoperfusion therapy. The newly developed extra-fine fibers are able to adsorb transforming growth 
factor (TGF)- $\beta$ one of the most potent immunosuppressors identified to date. TGF- $\beta$ has been isolated from many tumor cell lines and detected in plasma from tumor-bearing hosts. The degree of structural homology of TGF- $\beta$ among various mammals is $>97 \%$. Tumor cells secrete large amounts of TGF- $ß$. Besides its tumor-promoting effect, TGF- $\beta$ has been shown to suppress $\mathrm{T}$ cells by stimulating regulatory $\mathrm{T}$ cells or by direct inhibiting the differentiation of $\mathrm{T}$ cells into effector cytotoxic T lymphocytes (CTLs). Suppression of anti-tumor response by TGF- $\beta$ also involves inhibition of the maturation and antigen-presenting capacity of dendritic cells (14-18). In this study, rats bearing KDH-8 hepatocellular carcinoma cells, which secrete large amounts of TGF- $\beta$ were treated by direct hemoperfusion (DHP), using a column filled with ISA fibers (ISA column). We investigated the abilities of DHP treatment with an ISA column to remove TGF- $\beta$ from the blood, to restore cellular immune responses, and to prolong survival in this rat model. In addition, we studied the ability of ISA fibers to adsorb tumor-associated immunosuppressive cytokines such as TGF-ß, IL-6, and VEGF in samples of human body fluids.

\section{Materials and methods}

Animals and tumor cells. Male Wistar-King-Aptekman/Hok/ $\mathrm{Hkm}$ (WKAH/Hkm) rats (10-12 weeks old) were supplied by Shimizu Experimental Material Co., Ltd. (Kyoto, Japan). A transplantable hepatocellular carcinoma cell line, KDH-8, which was induced by 3'-methyl-4-dimethylaminoazobenzene in a WKAH/Hkm rat, was kindly provided by Dr Kazumasa Ogasawara (Second Department of Pathology, Shiga University of Medical Science). This cell line was maintained in RPMI-1640 (Nikken, Kyoto, Japan) supplemented with $50 \mu \mathrm{M}$ 2-mercaptoethanol, $50 \mu \mathrm{g} / \mathrm{ml}$ streptomycin, 50 units $/ \mathrm{ml}$ penicillin $\mathrm{G}$, and $10 \%$ fetal bovine serum (complete medium; CM).

Preparation of ISA fibers. ISA fibers were prepared as follows: Polystyrene-based composite fibers, comprised of polystyrene as the sheath component and polypropylene as the core component, were treated with an amidomethylating solution containing $\mathrm{N}$-methylol- $\alpha$-chloroacetamide, paraformaldehyde, sulfuric acid, and nitrobenzene for $2 \mathrm{~h}$ at $15^{\circ} \mathrm{C}$. The resulting chloroacetamidomethylated fibers were washed with methanol and allowed to react with $\mathrm{N}, \mathrm{N}$-dimethylhexylamine in dimethylformamide for $3 \mathrm{~h}$ at $80^{\circ} \mathrm{C}$. The fibers were thoroughly washed with isopropanol, followed by pyrogenfree distilled water. Extra-fine ISA fibers were thus obtained. The fibers were sterilized in an autoclave and kept at $4^{\circ} \mathrm{C}$ until use. Sterile cylindrical plastic columns with a capacity of $2.3 \mathrm{ml}$ were filled with $\sim 0.4 \mathrm{~g}$ of ISA fibers.

DHP treatment with ISA column in tumor-bearing rat model. On day $0, \mathrm{KDH}-8$ cells $\left(1 \times 10^{6}\right.$ cells $/$ rat $)$ were implanted subcutaneously into the back of WKAH/Hkm rats. On days 12-14 after tumor implantation, rats weighing 350 g underwent general anesthesia, and the left femoral artery and vein were cannulated surgically. DHP treatment was performed one time. The circuit used is shown in Fig. 1. Blood was withdrawn from the femoral artery, pumped via a peristaltic

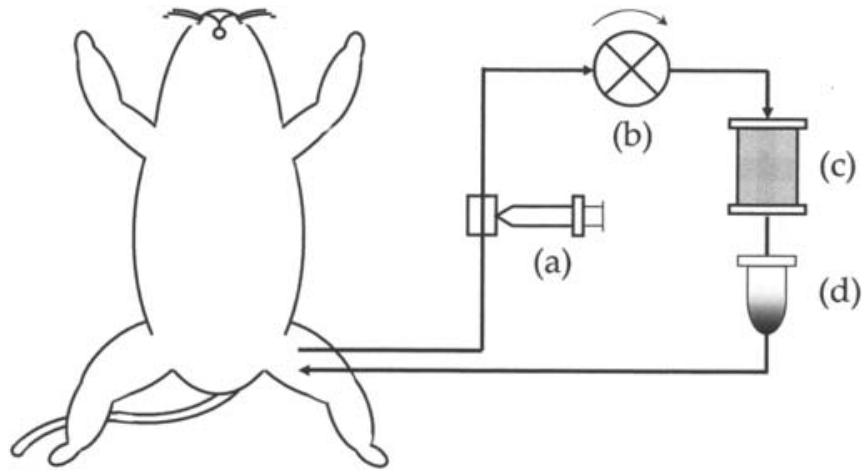

Figure 1. Schema of DHP treatment with an ISA column in the tumorbearing rats. (a) Heparin infusion (b) peristaltic pump (c) ISA column (d) air chamber. The priming volume was $3 \mathrm{ml}$; flow rate, $2 \mathrm{ml} / \mathrm{min}$. DHP, direct hemoperfusion; ISA, immunosuppressive substance adsorption.

pump at a flow rate of $2 \mathrm{ml} / \mathrm{min}$ in an upward direction through the column, and returned to the left femoral vein. DHP treatment was continuously performed for $1 \mathrm{~h}$. Before DHP, the circuit was primed with 1,000 units of heparin in $50 \mathrm{ml}$ of saline; during DHP, 100 units of heparin in total were infused continuously $(19,20)$. The rats were followed for survival and tumor growth, as compared with untreated rats. Tumor volume was calculated as follows: $\mathrm{A} \times \mathrm{B}^{2} \times 0.5$, where $\mathrm{A}$ is the length and $\mathrm{B}$ is the width of the tumor mass.

Cell line, mitogen, and chromogenic indicator for bioassay. Mv1Lu, a TGF- $\beta$-sensitive cell line derived from mink lung epithelial cells, was used to determine the bioactivity of TGF- $B$. This cell line was purchased from RIKEN Gene Bank (Tsukuba, Japan) and maintained in vitro in CM. Phytohemagglutinin (PHA) was purchased from Wako Pure Chemical Industries, Ltd. (Osaka, Japan). A water-soluble tetrazolium salt, 2-(2-methoxy-4-nitrophenyl)-3-(4-nitrophenyl)5-(2,4-disulfophenyl)-2H tetrazolium, monosodium salt (WST-8), which is useful for colorimetric cell viability assay, was purchased from Dojindo Laboratories (Kumamoto, Japan).

$T G F-\beta$ assays for rat serum. Serum levels of TGF- $\beta 1$ in tumorbearing rats were assayed by enzyme-linked immunoadsorbent assay (ELISA), using a human TGF- $\$ 1$ immunoassay system (TECHNE Corporation, Minneapolis, MN). This assay employs quantitative sandwich enzyme immunoassay techniques, in which 96-well microplates are pre-coated with TGF- $\beta$ soluble receptor type II, which binds TGF- 1 1. Each sample was pre-treated with acetic acid and neutralized with sodium hydroxide. The samples and standards of TGF- 11 were mounted into the wells and incubated for $2 \mathrm{~h}$ at room temperature. After washing away any unbound substances, an enzyme-linked polyclonal antibody specific for TGF- 11 was added to the wells to sandwich the TGF- $\beta 1$ immobilized during the first incubation. After a wash to remove any unbound antibody-enzyme reagent, a substrate solution was added to the wells. Color develops in proportion to the amount of TGF- $\beta 1$ bound in the initial step. Color development was stopped, and color intensity was measured on a microplate reader at $450 \mathrm{~nm}$, with a reference wavelength at $595 \mathrm{~nm}$. 


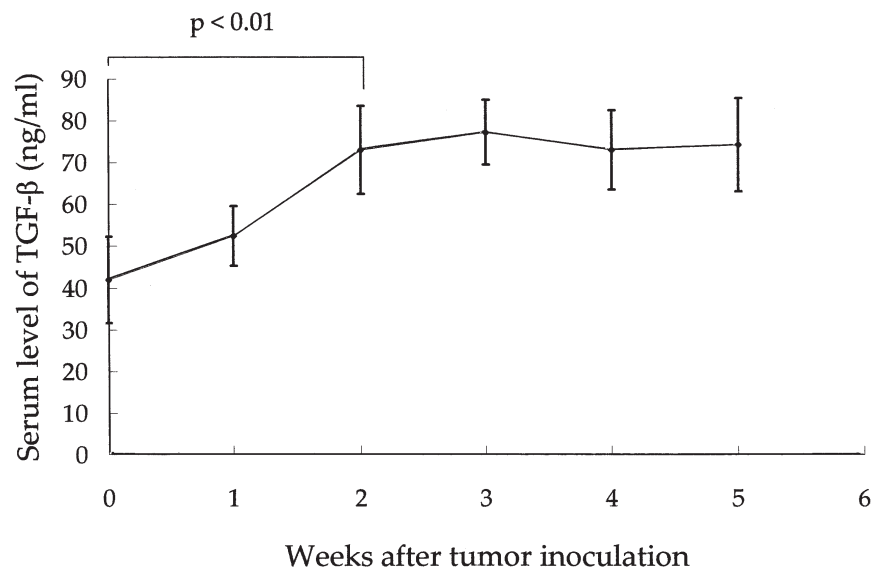

Figure 2. Serum levels of TGF- $\$ 1$ in tumor-bearing rats. Blood samples were obtained from KDH-8 tumor-bearing rats at various times. Tumorbearing rats were obtained by subcutaneous injection of $1 \times 10^{6} \mathrm{KDH}-8$ cells into the back. P was determined by unpaired Student's t-tests between serum levels of TGF- $\beta 1$ in KDH- 8 tumor-bearing rats at 0 and 2 weeks after tumor implantation; means $\pm \operatorname{SE}(n=4)$.

TGF- $\beta$ activity was measured by an in vitro bioassay based on the method described by Lucas et al, with some modifications (21). Briefly, Mv1Lu cells (1×104/well) were cultured with diluted samples in $200 \mu \mathrm{l} \mathrm{CM}$ in $96-$ well round-bottomed microplates. After incubation at $37^{\circ} \mathrm{C}$ and $5 \% \mathrm{CO}_{2}$ for $48 \mathrm{~h}$, WST-8 (10 $\mu \mathrm{l} /$ well) was added, and the plates were incubated for another $6 \mathrm{~h}$. The amount of surviving Mv1Lu cells was evaluated with the modified MTT method. WST- 8 produces a highly water-soluble formazan on cellular reduction. The absorbance of the formazan was measured on a microplate reader at $450 \mathrm{~nm}$, with a reference wavelength at $595 \mathrm{~nm}(22)$.

Proliferation assay of splenocytes. The proliferative responses of splenocytes to mitogenic stimulation in the presence of sera obtained from tumor-bearing rats before and after DHP treatment were assessed. The spleens were aseptically removed from normal rats. After removal of red blood cells by osmotic shock, the splenocytes were suspended in CM and cultured at $37^{\circ} \mathrm{C}$ and $5 \% \mathrm{CO}_{2}$ for $15 \mathrm{~h}$. The splenocytes $(180 \mu \mathrm{l}$; $1 \times 10^{6}$ cells $/ \mathrm{ml}$ ) were transferred to wells of 96-well roundbottomed microplates and supplemented with $20 \mu 1$ of sera from tumor-bearing rats (before and after DHP treatment) or normal rats plus $10 \mu \mathrm{l}$ PHA $(200 \mu \mathrm{g} / \mathrm{ml})$ (Sigma-Aldrich, Tokyo, Japan) $(23,24)$. After incubation for $72 \mathrm{~h}$, WST-8 (20 $\mu \mathrm{l} /$ well) was added to each well, and the plates were incubated for another $4 \mathrm{~h}$. The absorbance of the formazan converted from WST- 8 by surviving cells was measured on a microplate reader at $450 \mathrm{~nm}$, with a reference wavelength at $595 \mathrm{~nm}(22)$

Samples from patients with cancer. Four samples of human body fluids were collected from patients with metastatic cancer treated at the Department of Surgery, Kyoto Prefectural University of Medicine, Japan, between April 2003 and March 2004. One sample was serum from a patient with pancreatic cancer (male, age 62 years), and another was pleural effusion from a patient with esophageal cancer (male, age 70 years).
A

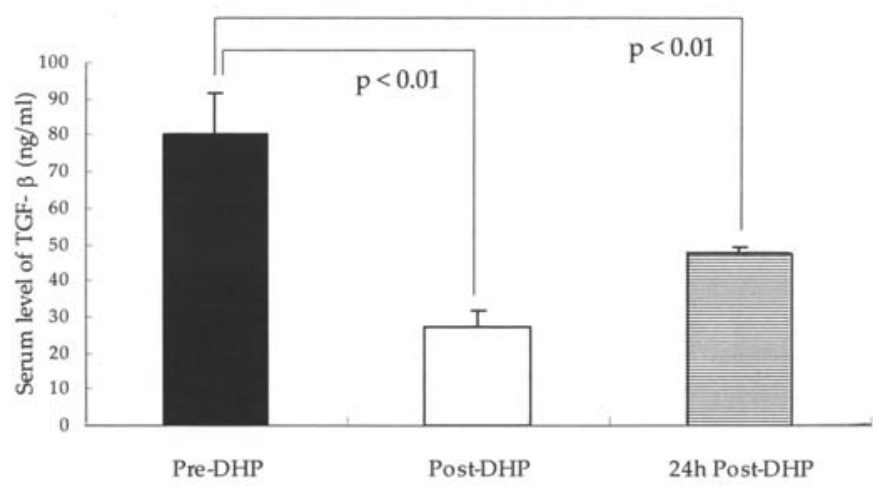

B

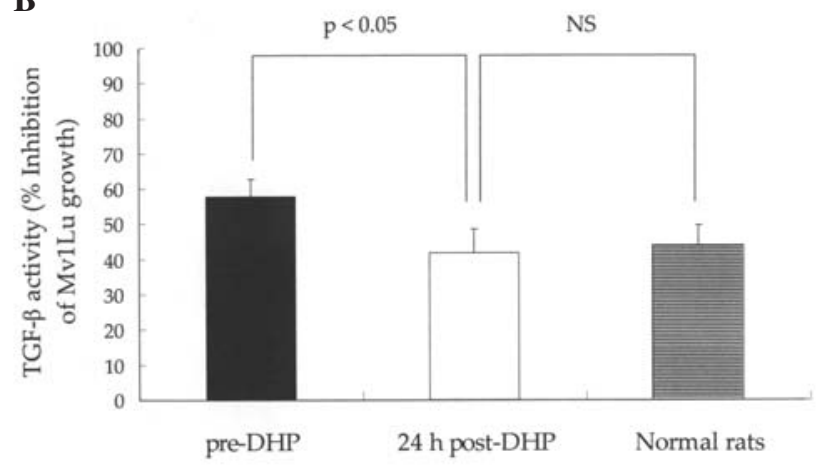

Figure 3. Reduction in serum levels and activities of TGF- $\beta$ in tumorbearing rats after DHP treatment. Tumor-bearing rats were prepared by the subcutaneous injection of $1 \times 10^{6} \mathrm{KDH}-8$ cells into the back. On day 14 after tumor implantation, rats were treated by DHP for $1 \mathrm{~h}$ with an ISA column. Blood samples were taken from the femoral arterial tap at the start of DHP (pre-DHP) and the end of DHP (post-DHP), and from the carotid arterial tap $24 \mathrm{~h}$ after DHP (24 h post-DHP). Blood samples of normal rats were also taken from the right carotid arterial tap as control. A, serum levels of TGF$\beta 1$ in tumor-bearing rats are presented as means \pm SE $(n=6)$. B, TGF- $\beta$ activities in the sera are presented as means $\pm \mathrm{SE}$ ( $\mathrm{n}=6$ in tumor bearing rats, $\mathrm{n}=3$ in normal rats). TGF- $\beta$ activity is expressed as $\%$ inhibition of Mv1Lu cell growth. The $\%$ inhibition was calculated as follows: $100 \times\left(1-A_{1} / A_{0}\right)$, where $A_{0}$ is the absorbance in culture without serum sample and $A_{1}$ is that in culture with serum sample; means $\pm \mathrm{SE}$. $\mathrm{P}<0.01,0.05$ was determined by unpaired Student's t-test.

The other two samples were ascites from patients with colon cancer and scirrhous gastric cancer, respectively (male, age 65 years and female, age 58 years). Pleural effusion and ascites were aseptically collected and mixed with 5 units $/ \mathrm{ml}$ heparin.

Tumor-associated cytokine assay in human samples. Human samples were assayed for concentrations of tumor-associated cytokines (IL-6, IL-10, TGF-31, and VEGF) by SRL Inc. (Tokyo, Japan), using the following commercially available kits for ELISA: Human IL-6 CLEIA kit from Fujirebio Inc. (Tokyo, Japan); Human IL-10 EASIA kit from BioSource International Inc. (Camarillo, CA); and Quantikine Human TGF- 11 and VEGF immunoassays from R \& D Systems (Minneapolis, MN). The samples were treated by passing through a syringe filled with $0.5 \mathrm{~g}$ of ISA fibers at a rate of $0.5 \mathrm{ml} / \mathrm{min}$. Cytokine concentrations were measured before and after in vitro treatment with ISA fibers.

Statistical analysis. All data except for survival are expressed as means \pm standard error (SE). For comparison of two groups, 


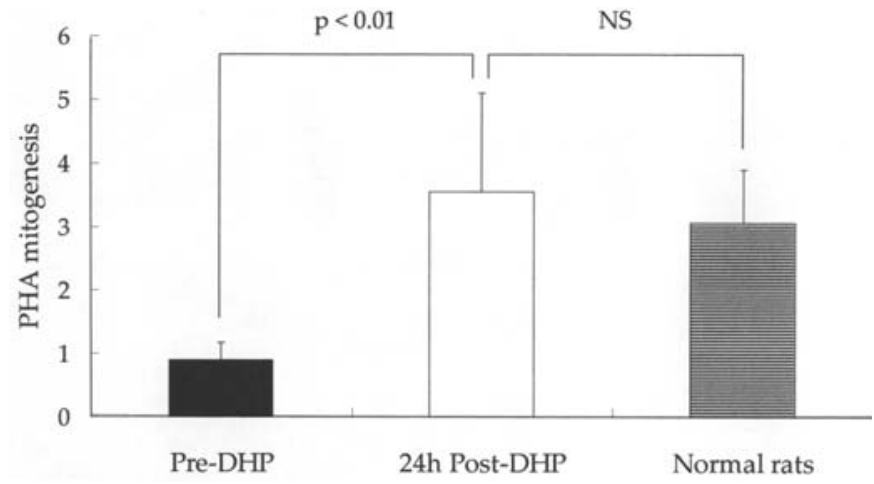

Figure 4. Restoration of PHA-induced proliferation of splenocytes in the presence of sera from tumor-bearing rats treated by DHP. Tumor-bearing rats were prepared by the subcutaneous injection of $1 \times 10^{6} \mathrm{KDH}-8$ cells into the back. On day 14 after tumor implantation, rats were treated by DHP with an ISA column for $1 \mathrm{~h}$. Blood samples were taken from the left femoral arterial tap at the start of DHP (pre-DHP) and from the right carotid arterial tap $24 \mathrm{~h}$ after DHP ( $24 \mathrm{~h}$ post-DHP) in tumor-bearing rats. Blood samples of normal rats were taken from the right carotid arterial tap. PHA-induced proliferation of splenocytes from normal rats in the presence of sera from tumor-bearing rats or normal rats was calculated as follows: $\left(A_{1}-A_{0}\right) /\left(A_{2}-A_{0}\right)$, where $\mathrm{A}_{0}$ is the absorbance in $\mathrm{CM}, \mathrm{A}_{1}$ is that in culture containing the sample with PHA, and $\mathrm{A}_{2}$ is that in culture containing the sample without PHA. means $\pm \operatorname{SE}(n=6$; tumor bearing rats, $n=3$; normal rats), $\mathrm{P}<0.01$ was determined by unpaired Student's t-test.

Student's t-test was used. The level of significance was set at $\mathrm{p}<0.05$. The Kaplan-Meier method in combination with logrank tests was used to analyze survival data.

\section{Results}

Serum levels of TGF- $\beta 1$ in tumor-bearing rats. On day 0 , $\mathrm{KDH}-8$ cells $\left(1 \times 10^{6}\right.$ cells/rat $)$ were implanted s.c. into the back of WKAH/Hkm rats. Tumors became palpable within 1-2 weeks after tumor implantation, progressed rapidly in 3-4 weeks, and grew extremely rapidly in 5-6 weeks, until the rats died of cachexia.

Serum levels of TGF- $B 1$ in tumor-bearing rats increased significantly in association with tumor growth until 3 weeks after tumor implantation and then decreased slightly or reached a plateau (Fig. 2). In contrast, there were no significant elevations in serum levels of IL-6 or VEGF until 4 weeks after tumor implantation (data not shown).

Reductions in serum levels and activities of TGF- $\beta$ in tumorbearing rats after DHP treatment. We examined whether DHP treatment with the use of an ISA column could reduce the elevated serum levels of TGF- $\beta$ in KDH-8 tumor-bearing rats. After DHP treatment, serum TGF- $\beta 1$ levels decreased significantly. Mean serum TGF- $\beta 1$ levels before DHP, immediately after DHP, and $24 \mathrm{~h}$ after DHP were $80.0 \pm 11.7$, $27.2 \pm 4.7$, and $47.3 \pm 1.9$, respectively ( $\mathrm{p}<0.01$ : pre-DHP vs. post-DHP, $24 \mathrm{~h}$ post-DHP, Fig. 3A). We also examined the change in serum TGF- $\beta$ activities in tumor-bearing rats. DHP treatment significantly reduced the serum TGF- $\beta$ activity of tumor-bearing rats $24 \mathrm{~h}$ after treatment $(\mathrm{p}<0.05$, Fig. $3 \mathrm{~B})$.

Restoration of PHA-induced proliferation of splenocytes in the presence of sera from tumor-bearing rats treated by DHP. To

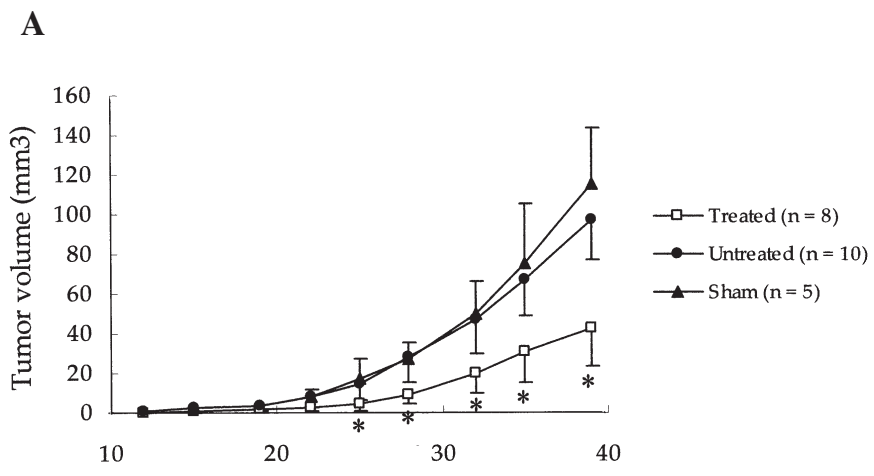

Days after tuomr implantation

B

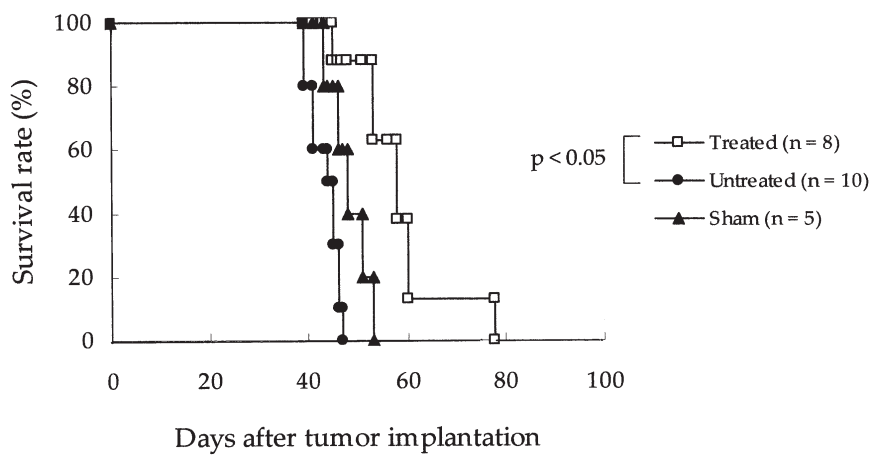

Figure 5. Antitumor effects of DHP with an ISA column in tumor-bearing rats. Tumor-bearing rats were obtained by the subcutaneous injection of $1 \times 10^{6}$ viable KDH- 8 cells into the back. These rats were divided into the following 3 groups: treated group, rats were treated by DHP for $1 \mathrm{~h}$ on day 12 after tumor implantation $(\square)$; sham group, rats were treated with an empty column $(\mathbf{\Lambda})$; untreated group, rats not treated $(\bullet)$. A, individual growth curves of KDH-8 tumor. Tumor growth was monitored twice a week by caliper measurement of tumor length and width from the time of DHP treatment and expressed as mean tumor volume $\left(\mathrm{mm}^{3}\right)$; Bar, SE. ${ }^{*} \mathrm{P}<0.01$ as determined by unpaired Student's t-tests between the treated group and the untreated group. B, survival curves of KDH-8 tumor-bearing rats recorded as the percentage of surviving animals on a given day. $\mathrm{P}<0.05$ was determined by the Kaplan-Meier method in combination with log-rank tests between the treated group and the untreated group.

investigate the effect of DHP treatment on cellular immune responses, the proliferative responses of splenocytes from normal rats to mitogenic stimulation in the presence of sera obtained from tumor-bearing rats before and after DHP were assessed. Restoration of PHA-induced proliferation of splenocytes was observed after DHP treatment. The activity of the sera from tumor-bearing rats after DHP was nearly equal to that of the sera from normal rats (Fig. 4). These results suggest that cellular immune responses abolished in the presence of sera from tumor-bearing hosts can be restored by DHP treatment.

Effects of DHP with ISA column on tumor growth and survival. We examined the antitumor effects of DHP with an ISA column in KDH-8 tumor-bearing rats. A single DHP treatment was carried out on day 12 after tumor implantation. Tumor growth in the DHP-treated group was significantly slower than that in the untreated group $(\mathrm{P}<0.01$, on days $25,28,32,35$, and 39, Fig. 5A). The mean survival times of the DHP-treated 

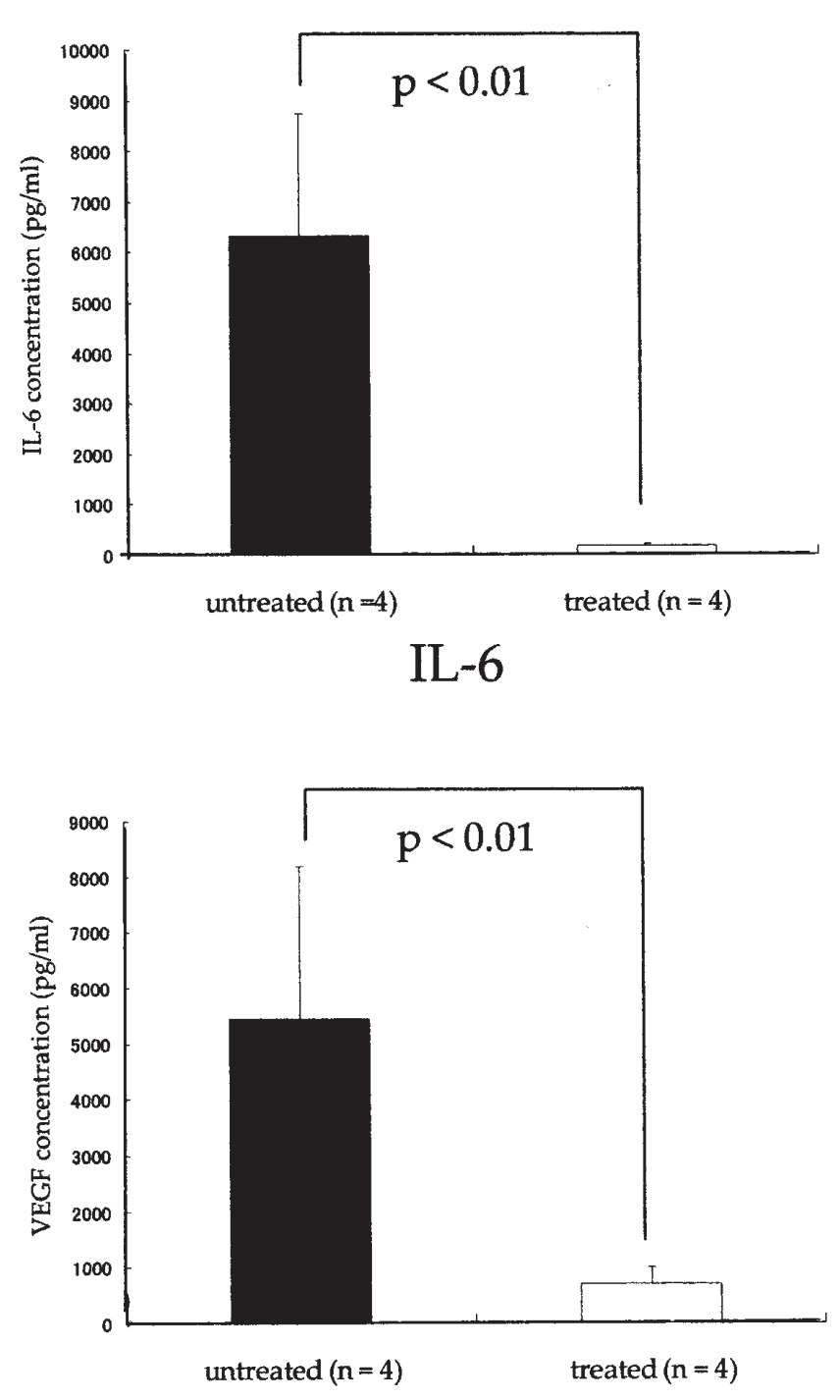

VEGF

group and the untreated group were 58.1 \pm 9.5 and 43.3 \pm 3.0 days, respectively. Further survival analysis by the KaplanMeier method showed that the differences in survival times were significant between the 2 groups $(\mathrm{P}<0.05$, Fig. $5 \mathrm{~B})$. These results show that a single DHP treatment suppresses tumor growth and thus prolongs the survival of tumor-bearing rats. In contrast, there were no significant differences in tumor growth or mean survival times between the untreated group and sham group.

In vitro adsorption of tumor-associated cytokines in human samples by ISA fibers. To assess the abilities of ISA fibers to adsorb tumor-associated cytokines, we determined the concentrations of four cytokines, IL-6, TGF- 31 , IL-10, and VEGF, in the four samples of body fluids obtained from patients with metastatic cancer. Cytokine concentrations were measured before and after in vitro treatment with ISA fibers. In all four untreated samples, appreciable concentrations of IL-6, TGF- 11 , and VEGF were detected, whereas IL-10 was not detected (data not shown). The concentrations of IL-6, TGF- $\$ 1$, and VEGF in each treated sample were significantly lower than those in each untreated sample. Mean concentrations in the treated group and the untreated group were

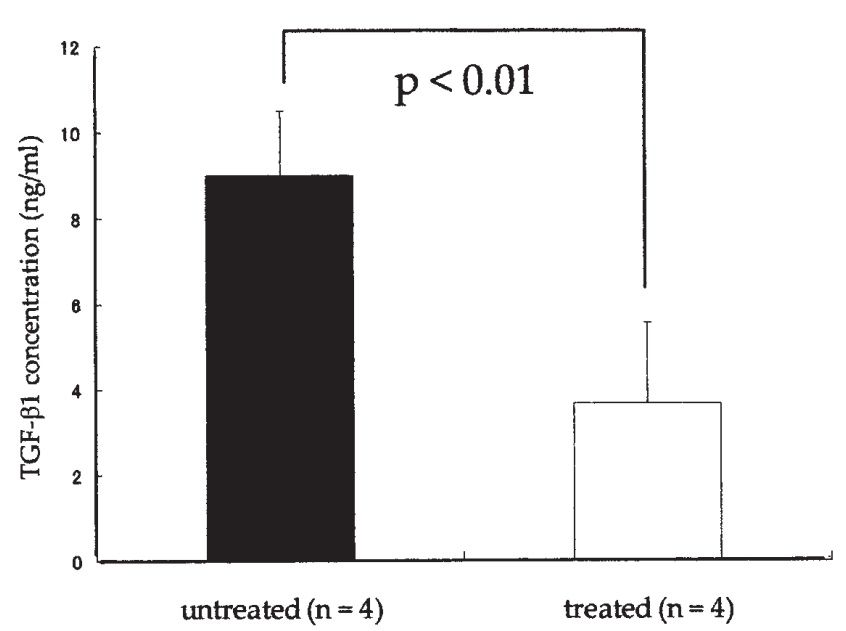

TGF- $\beta 1$

Figure 6. In vitro adsorption of tumor-associated cytokines in human samples by ISA fibers. Four human samples from patients with metastatic cancer were divided into two groups: treated group, samples were treated by passing through a syringe filled with $0.5 \mathrm{~g}$ of ISA fibers at a rate of $0.5 \mathrm{ml} /$ min; untreated group, samples were not treated. A, IL-6; B, TGF- 3 ; C, VEGF. The concentrations of these cytokines were measured in each group by ELISA. means $\pm S E(n=4) . P<0.01$ was determined by unpaired Student's t-test.

respectively $7,090 \pm 2,261$ and $159 \pm 29 \mathrm{pg} / \mathrm{ml}$ for IL-6; $9.00 \pm 1.51$ and $3.69 \pm 1.88 \mathrm{ng} / \mathrm{ml}$ for TGF- $\beta 1$; and $5,454 \pm 2,742$ and $703 \pm 294 \mathrm{pg} / \mathrm{ml}$ for VEGF $(\mathrm{p}<0.01$ : treated vs. untreated, Fig. 6). These results suggested that DHP treatment with an ISA column reduces the elevated serum levels of tumorassociated cytokines such as IL-6, TGF- 1 , and VEGF in tumor-bearing hosts.

\section{Discussion}

This study showed that our newly developed ISA fibers adsorbed tumor-associated immunosuppressive cytokines in vitro and demonstrated that DHP treatment with an ISA column adsorbed TGF- 31 , particularly in the blood of KDH-8 tumor-bearing rats. Moreover, we showed that DHP treatment significantly suppressed tumor growth and prolonged survival of the rats.

We previously developed a hemoperfusion column (Toraymyxin) containing porous cellulose beads covalently bound to a surface ligand comprised of a hydrophobic organic compound with a hexadecyl alkyl chain. This column was designed to remove bacterial toxins and cytokines in diseases associated with elevated levels of inflammatory cytokines, such as 


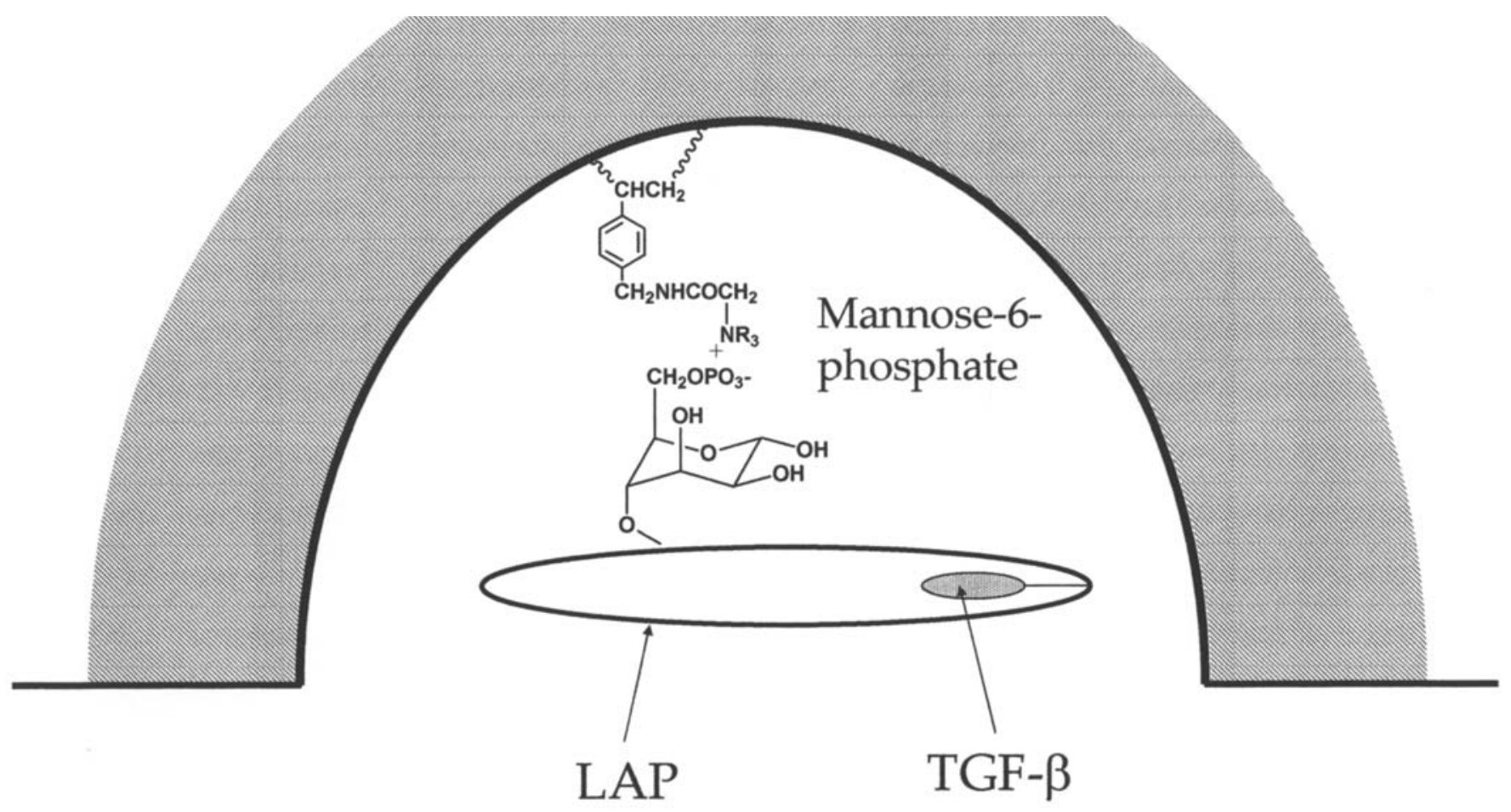

Figure 7. Mechanism by which ISA fibers adsorb TGF-ß. ISA fibers have a strong affinity for anionic proteins because they structurally are a porous solid and chemically have a quaternary ammonium group. TGF- $\beta$ exists in latent form in blood. Latent TGF- $\beta$ consists of active TGF- $\beta$ and latency associated peptide (LAP), a glycoprotein with polysaccharides connected by mannose-6-phosphate in LAP.

bacterial endotoxemia (13). Hemoperfusion therapy with this column has been proven to efficiently adsorb IL-1B, IL-6, and 32-microglobulin. Oda et al have demonstrated the enhanced adsorbent properties of a column containing cellulose beads cross-linked with hexamethylene-di-isocyanate, which efficiently adsorbs cytokines such as TNF- $\alpha$, IL-6, and IL-10 (25). Immunoadsorption columns containing Toraymyxin are now being evaluated in clinical trials.

In this study, we examined the potential of using DHP treatment with an ISA column for cancer therapy. Since TGF- $\beta$ plays a critical role in tumor-induced immunosuppression (18), its removal from cancer-bearing hosts may promote tumor eradication as well as improve patients' quality of life. Indeed, several approaches have previously been used to reverse the effects of elevated levels of TGF- $B$. Systemic administration of TGF- $\beta$ neutralizing antibodies is one example that has been already tested in several clinical trials (26-28). Inhibition of TGF- $\beta$ production by the use of TGF- $\beta$ antisense has been experimentally shown to be a promising approach in vitro as well as in vivo $(29,30)$. However, the clinical use of these materials may be limited by the formation of anti-idiotype antibodies and the very short half-life of antisense oligos in serum $(31,32)$. To our knowledge, trials of hemoperfusion therapy targeting TGF- $\beta$ have not been performed thus far. Of the three isoforms of TGF- $\beta$ (TGF- $\beta 1,-\beta 2$, and - $\beta 3$ ), TGF$B 1$ is the most abundant and universally expressed. Since most TGF- $\beta 1$ exists in a latent form, the column should be designed to adsorb latent TGF- $\beta 1$. Latent TGF- 11 comprises active TGF- $B 1$ and latency associated peptide (LAP), a glycoprotein with polysaccharides connected by mannose6-phosphate in LAP (4). The molecular weight of latent
TGF- $\beta 1$ is $\sim 100 \mathrm{kDa}$, while that of IL-6 or $\beta 2$-microglobulin is $<30 \mathrm{kDa}$. The column was prepared by amidomethylation. The endotoxin-removing fibers, Toraymyxin, were synthesized to target high molecular weight proteins. First, the polypropylene-reinforced polystyrene fibers were amidomethylated with $\mathrm{N}$-hydroxymethyl-2-chloroacetamide to obtain 2-chloroacetamidemethylated polystyrene fibers, porous carrier fibers that are available for DHP therapy. These fibers can immobilize various ligands. Second, the carrier fibers were treated with $\mathrm{N}, \mathrm{N}$-dimethyl-hexylamine to produce $\mathrm{N}, \mathrm{N}$ dimethyl-hexylammono-acetamidomethylated polystyrene fibers (ISA fibers). The quaternary ammonium group is hydrophilic and anionic; ISA fibers therefore have a strong affinity for latent TGF- $\beta 1$. ISA fibers have a porous structure resulting from amidomethylation, similar to 'Toraymyxin'. Unlike 'Toraymyxin', the ligand of ISA fibers has been switched from polymixin to an $\mathrm{N}, \mathrm{N}$-dimethyl-hexylammono group, and the shape has changed from a net to a direct fabrication (DF). In contrast to net-like fibers, DF fibers do not adhere together, thereby avoiding a decrease in surface area during amidomethylation. As shown in Fig. 6, ISA fibers have large adsorption capacities for IL-6, VEGF, and LAP, which has a molecular weight of $45 \mathrm{kDa}$. ISA fibers adsorb LAP because the pores are large enough for LAP to enter, and that there is an ionic interaction between the mannose-6-phosphate residue on LAP and the $\mathrm{N}, \mathrm{N}$-dimethyl-hexylammono group on the fibers (Fig. 7).

$\mathrm{KDH}-8$ is a rat hepatocellular carcinoma cell line that secretes large amounts of TGF- $\beta$. The resulting high levels of TGF- $\beta$ contribute to the malignant properties of this line, mainly by suppressing immune functions of the host. Yuan 
et al reported that inhibition of TGF- $\beta$ production by $\mathrm{KDH}-8$ cells restores host immune responses and consequently suppresses tumor growth (33). In our experiments, serum TGF- $\$ 1$ levels in the KDH-8 tumor-bearing rats increased with time after inoculation from $40 \mathrm{ng} / \mathrm{ml}$ to as high as $70 \mathrm{ng} / \mathrm{ml}$ (Fig. 1). DHP treatment with an ISA column markedly reduced the elevated serum levels of TGF- $\$ 1$ in the tumor-bearing rats (Fig. 3A). Moreover, PHA-mitogenesis of the rat splenocytes in the presence of sera from DHP-treated tumor-bearing rats was nearly the same as that in the presence of sera from normal rats (Fig. 4). In contrast, PHA-mitogenesis was markedly suppressed in the presence of sera from non-treated tumorbearing rats. These results indicate that the sera from tumorbearing rats were immunosuppressive and suggest that immune activity can be restored by DHP treatment with an ISA column. We further showed that tumor progression was suppressed by DHP treatment with an ISA column. Consistent with the results of previous studies, our approach to reduce increased serum TGF-ß1 levels by DHP seemed to enhance immune functions and produce anti-tumor effects in tumor-bearing rats.

Using samples from patients with advanced cancer, we found that ISA fibers sufficiently adsorb not only TGF- $\beta$, but also IL-6 and VEGF in vitro (Fig. 6). This concomitant effect may further enhance the response to treatment in patients with cancer, since tumor-induced immunosuppression is not regulated by TGF- $\beta$ alone. Further studies of hemoperfusion therapy with ISA columns should examine detailed immune responses, including the activities of effector cells such as T cells, NK cells, and NKT cells, as well as the activities of antigen-presenting cells such as dendritic cells after treatment.

In conclusion, hemoperfusion therapy with an ISA column may be a useful and cost-effective approach to reducing levels of immunosuppressive cytokines, as compared with other techniques for extracorporeal circulation, such as plasma exchange. Whether immunosuppressive cytokine levels are efficiently reduced in tumor tissues as well as in serum remains a topic for future research. To achieve better outcomes, development of a hybrid column containing ISA fibers and pokeweed mitogen (PWM) (34), which has lymphocyteactivation properties, is currently underway.

\section{Acknowledgements}

This work was supported by a 2003 Grant for R\&D for practical application utilizing the matching fund method from the New Energy and Industrial Technology Development Organization, Japan.

\section{References}

1. Ashizawa T, Okada R, Suzuki Y, Takagi M, Yamazaki T, Sumi T, Aoki T, Ohnuma $S$ and Aoki T: Clinical significance of interleukin-6 (IL-6) in the spread of gastric cancer: role of IL-6 as a prognostic factor. Gastric Cancer 8: 124-131, 2005.

2. Ohm JE, Gabrilovich DI, Sempowski GD, Kisseleva E, Parman KS, Nadaf S and Carbone DP: VEGF inhibits T-cell development and may contribute to tumor-induced immune suppression. Blood 101: 4878-4886, 2003.

3. Miyazono K, Suzuki H and Imamura T: Regulation of TGF-beta signaling and its roles in progression of tumors. Cancer Sci 94: 230-234, 2003.
4. Elliott RL and Blobe GC: Role of transforming growth factor beta in human cancer. J Clin Oncol 23: 2078-2093, 2005.

5. Wojtowicz-Praga S: Reversal of tumor-induced immunosuppression: a new approach to cancer therapy. J Immunother 20: 165-177, 1997.

6. Saito H, Tsujitani S, Oka S, Kondo A, Ikeguchi M, Maeta M and Kaibara N: The expression of transforming growth factorbeta1 is significantly correlated with the expression of vascular endothelial growth factor and poor prognosis of patients with advanced gastric carcinoma. Cancer 86: 1455-1462, 1999.

7. Tani T, Numa K, Hanasawa $K$ and Kodama M: Blood purification therapy in cancer treatment. Ther Apher 2: 182-184, 1998.

8. Browne O, Bell J, Holland PD and Thornes RD: Letter: Plasmapheresis and immunostimulation. Lancet 2: 96, 1976.

9. Hersey P, Isbister J, Edwards A, Murray E, Adams E, Biggs J and Milton GW: Antibody-dependent cell-mediated cytotoxicity against melanoma cells induced by plasmapheresis. Lancet 1: 825-828, 1976.

10. Micksche M, Colot M, Kokoschka EM, Moser K and Rainer H: Plasmapheresis in patients with advanced malignant disease: a pilot study. Oncology 39: 146-151, 1982.

11. Israel L, Edelstein R, Mannoni P and Radot E: Plasmapheresis and immunological control of cancer. Lancet 2: 642-643, 1976.

12. Israel L, Edelstein R, Mannoni P, Radot E and Greenspan EM: Plasmapheresis in patients with disseminated cancer: clinical results and correlation with changes in serum protein. The concept of 'nonspecific blocking factors'. Cancer 40: 3146-3154, 1977.

13. Hanasawa K, Tani T, Oka T, Yoshioka T, Endo Y, Horisawa M, Nakane Y, Kodama M, Teramoto K and Nishiumi S: A new treatment for endotoxemia with direct hemoperfusion by polymyxin immobilized fiber. Ther Apher 4: 142-145, 2000.

14. Kobie JJ, Wu RS, Kurt RA, Lou S, Adelman MK, Whitesell LJ, Ramanathapuram LV, Arteaga CL and Akporiaye ET: Transforming growth factor beta inhibits the antigen-presenting functions and antitumor activity of dendritic cell vaccines. Cancer Res 63: 1860-1864, 2003.

15. Somasundaram R, Jacob L, Swoboda R, Caputo L, Song H, Basak S, Monos D, Peritt D, Marincola F, Cai D, Birebent B, Bloome E, Kim J, Berencsi K, Mastrangelo M and Herlyn D: Inhibition of cytolytic $\mathrm{T}$ lymphocyte proliferation by autologous $\mathrm{CD} 4^{+} / \mathrm{CD} 25^{+}$regulatory $\mathrm{T}$ cells in a colorectal carcinoma patient is mediated by transforming growth factor-beta. Cancer Res 62: 5267-5272, 2002

16. Yamagiwa S, Gray JD, Hashimoto $S$ and Horwitz DA: A role for TGF-beta in the generation and expansion of $\mathrm{CD} 4{ }^{+} \mathrm{CD} 25^{+}$ regulatory $\mathrm{T}$ cells from human peripheral blood. J Immunol 166: 7282-7289, 2001.

17. Gorelik L and Flavell RA: Transforming growth factor-beta in T-cell biology. Nat Rev Immunol 2: 46-53, 2002.

18. de Visser KE and Kast WM: Effects of TGF-beta on the immune system: implications for cancer immunotherapy. Leukemia 13: 1188-1199, 1999.

19. Courtney JM, Murphy SM, Robertson LM, Ryan CJ and Tighe BJ: Examination of particulate macroporous hydrogels in an extracorporeal rat haemoperfusion model. J Biomater Sci Polym Ed 10: 1063-1077, 1999.

20. Fenwick P, Ryan C, Sriskandan S and Cohen J: Application of a rat model of streptococcal shock to evaluate on-line hemoperfusion and removal of circulating superantigens. Crit Care Med 31: 171-178, 2003

21. Lucas C, Fendly BM, Mukku VR, Wong WL and Palladino MA: Generation of antibodies and assays for transforming growth factor beta. Methods Enzymol 198: 303-316, 1991.

22. Tominaga $H$, Ishiyama M, Ohseto $F$, Sasamoto K, Hamamoto T and Suzuki K: A water-soluble tetrazolium salt useful for colorimetric cell viability assay. Anal Commun 36: 47-50, 1999.

23. Korzeniewski $\mathrm{C}$ and Callewaert DM: An enzyme-release assay for natural cytotoxicity. J Immunol Methods 64: 313-320, 1983.

24. Tsai YC and Won SJ: Effects of tramadol on T lymphocyte proliferation and natural killer cell activity in rats with sciatic constriction injury. Pain 92: 63-69, 2001.

25. Oda S, Hirasawa H, Shiga H, Nakanishi K, Matsuda K, Nakamura M, Ikeda $\mathrm{H}$ and Sakai M: Cytokine adsorptive property of various adsorbents in immunoadsorption columns and a newly developed adsorbent: an in vitro study. Blood Purif 22: 530-536, 2004. 
26. Blottiere HM, Steplewski Z, Herlyn D and Douillard JY: Human anti-murine immunoglobulin responses and immune functions in cancer patients receiving murine monoclonal antibody therapy. Hum Antibodies Hybridomas 2: 16-25, 1991.

27. Grossbard ML, Press OW, Appelbaum FR, Bernstein ID and Nadler LM: Monoclonal antibody-based therapies of leukemia and lymphoma. Blood 80: 863-878, 1992.

28. Greenaway S, Henniker AJ, Walsh M and Bradstock KF: A pilot clinical trial of two murine monoclonal antibodies fixing human complement in patients with chronic lymphatic leukaemia. Leuk Lymphoma 13: 323-331, 1994.

29. Huang F, Newman E, Theodorescu D, Kerbel RS and Friedman E: Transforming growth factor beta 1 (TGF beta 1) is an autocrine positive regulator of colon carcinoma U9 cells in vivo as shown by transfection of a TGF beta 1 antisense expression plasmid. Cell Growth Differ 6: 1635-1642, 1995.

30. Marzo AL, Fitzpatrick DR, Robinson BW and Scott B. Antisense oligonucleotides specific for transforming growth factor beta 2 inhibit the growth of malignant mesothelioma both in vitro and in vivo. Cancer Res 57: 3200-3207, 1997.
31. Winter $\mathrm{G}$ and Harris WJ: Humanized antibodies. Immunol Today 14: 243-246, 1993.

32. Agrawal S, Temsamani J, Galbraith W and Tang J: Pharmacokinetics of antisense oligonucleotides. Clin Pharmacokinet 28: 7-16, 1995.

33. Yuan L, Kuramitsu Y, Li Y, Kobayashi M and Hosokawa M: Restoration of interleukin-2 production in tumor-bearing rats through reducing tumor-derived transforming growth factor beta by treatment with bleomycin. Cancer Immunol Immunother 41: 355-362, 1995.

34. Numa K, Tani T and Kodama M: Trial of anticancer immunotherapy with immobilized pokeweed mitogen: immunotherapy by extracorporeal circulation. Cancer Immunol Immunother 32: $125-130,1990$. 